\title{
Thermally assisted ordering in Mott insulators
}

\author{
Hunter Sims, ${ }^{1}$ Eva Pavarini, ${ }^{2,3}$ and Erik Koch ${ }^{1,2,3, *}$ \\ ${ }^{1}$ Computational Materials Science, German Research School for Simulation Sciences, 52425 Jülich, Germany \\ ${ }^{2}$ Institute for Advanced Simulation, Forschungszentrum Jülich, 52425 Jülich, Germany \\ ${ }^{3}$ JARA High-Performance Computing, 52425 Jülich, Germany
}

(Received 16 November 2016; revised manuscript received 19 July 2017; published 8 August 2017)

\begin{abstract}
Landau theory describes phase transitions as the competition between energy and entropy: The ordered phase has lower energy, while the disordered phase has larger entropy. When heating the system, ordering is reduced entropically until it vanishes at the critical temperature. This picture implicitly assumes that the energy difference between the ordered and disordered phases does not change with temperature. We show that for orbital ordering in the Mott insulator $\mathrm{KCuF}_{3}$, this assumption fails qualitatively: entropy plays a negligible role, while thermal expansion energetically stabilizes the orbitally ordered phase to such an extent that no phase transition is observed. To understand this strong dependence on the lattice constant, we need to take into account the Born-Mayer repulsion between the ions. It is the latter, and not the Jahn-Teller elastic energy, which determines the magnitude of the distortion. This effect will be seen in all materials where the distortion expected from the Jahn-Teller mechanism is so large that the ions would touch. Our mechanism explains not only the absence of a phase transition in $\mathrm{KCuF}_{3}$, but even suggests the possibility of an inverted transition in closed-shell systems, where the ordered phase emerges only at high temperatures.
\end{abstract}

DOI: 10.1103/PhysRevB.96.054107

Mott insulators with orbital degrees of freedom often exhibit orbitally ordered phases [1]. There are two established explanations for this: (i) electron-phonon coupling induces cooperative Jahn-Teller distortions [2] that lead to orbital ordering or (ii) Kugel-Khomskii superexchange [3] gives rise to orbital order that leads to a cooperative lattice distortion. Since both mechanisms tend to result in the same type of ordering, identifying which one drives it is a "chicken-andegg problem" [4]. Even though they strongly differ, these two mechanisms have one fundamental aspect in common: Their hallmark is a conventional Landau-type second-order transition [5,6] between a low-temperature ordered structure and a symmetric high-temperature phase [7]. This implies that the distortion, which acts as the order parameter, goes to zero at the transition temperature. Here we show that in $\mathrm{KCuF}_{3}$, this conventional picture fails qualitatively: The Jahn-Teller mode is so soft that the distortion is determined by the Born-Mayer repulsion of the ions. As a consequence, the distortion and the corresponding gain in energy increase with lattice constant, so that, via thermal expansion, the order parameter increases with temperature. We anticipate that this thermally assisted ordering can operate even in closed-shell systems. This would result in an inverted Landau transition, with symmetry breaking above a critical temperature. These surprising conclusions are based on very general arguments. We thus expect that similar effects will play a key role in other ordering phenomena of totally different nature.

Following the seminal work of Kugel and Khomskii [3], the fluoride $\mathrm{KCuF}_{3}$ is considered the prototype of an orbitally ordered material. Its structure [8], shown in Fig. 1, derives from a cubic perovskite with $\mathrm{Cu}$ in $d^{9}$ configuration with one hole in the $e_{g}$ orbital surrounded by an octahedron of fluoride ions in a cage of potassium ions. The actual crystal shows a tetragonal compression, slightly lifting the $e_{g}$ degeneracy. The fluoride

\footnotetext{
*e.koch@fz-juelich.de
}

ions in the $a-b$ plane move along the lines connecting the $\mathrm{Cu}$ ions such that long $(\ell)$ and short $(s)$ bonds alternate in the $x$ and $y$ directions, leading to a cooperative Jahn-Teller distortion and a competing splitting of the $e_{g}$ orbital. The distortion pattern also alternates in the $z$ direction, resulting in an antiferrodistortive orbital ordering. The tetragonal distortion is parametrized by $c / a \sqrt{2}$ and the Jahn-Teller distortion by $\delta=(\ell-s) / a \sqrt{2}$.

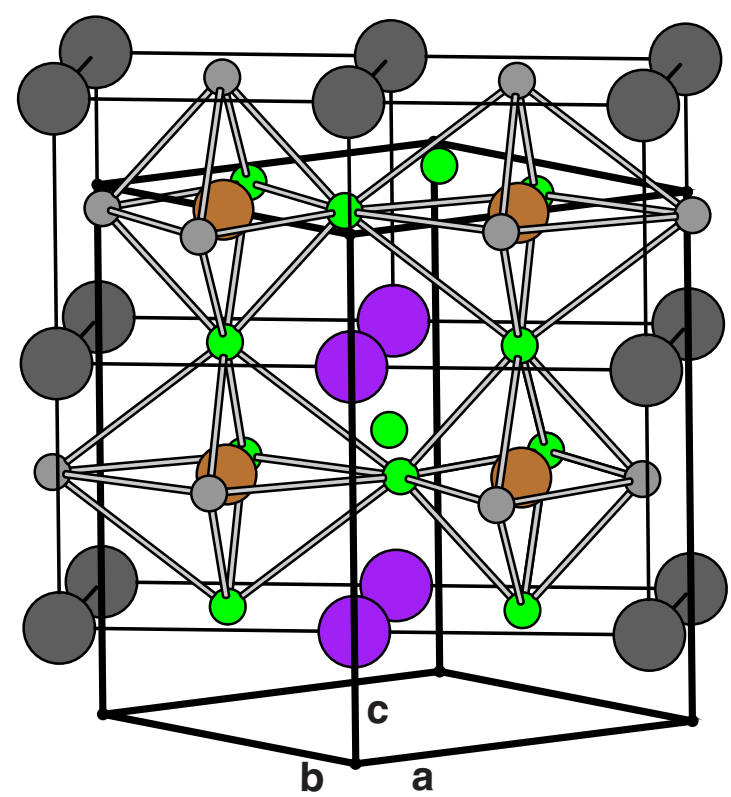

FIG. 1. Crystal structure of $\mathrm{KCuF}_{3}$. Inequivalent atoms inside the $I 4 / \mathrm{mcm}$ unit cell (thick black lines) are shown in color $(\mathrm{Cu}$ : brown; $\mathrm{F}$ : green; $\mathrm{K}$ : violet). The additional atoms in gray show the pseudocubic setting in which the network of corner-sharing octahedra becomes apparent. The pseudocubic axes are defined as $\mathbf{x}=(\mathbf{a}+\mathbf{b}) / 2$, $\mathbf{y}=(-\mathbf{a}+\mathbf{b}) / 2$, and $\mathbf{z}=\mathbf{c} / 2$. For clarity, lattice distortions are exaggerated twofold. 


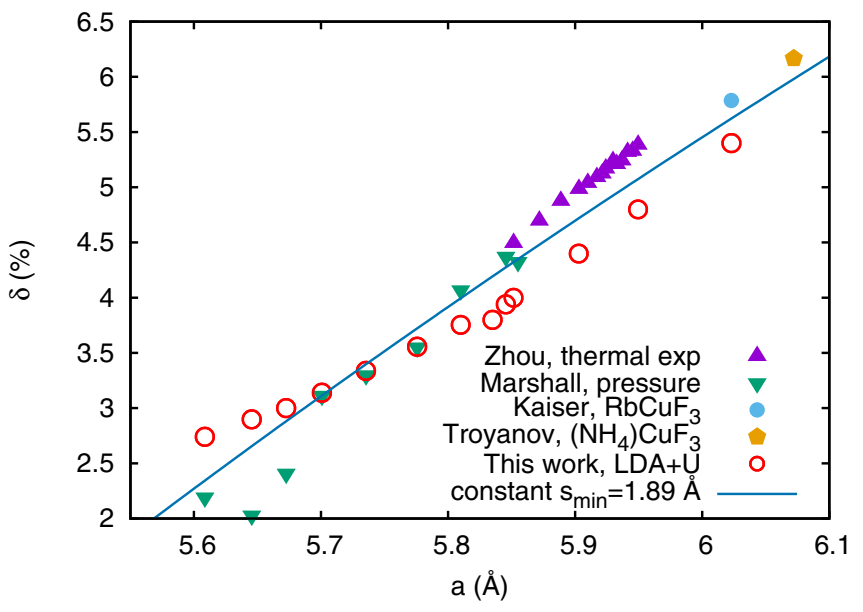

FIG. 2. Distortion parameter $\delta$ as a function of lattice constant $a$ in thermally expanding $\mathrm{KCuF}_{3}$ [12] and under hydrostatic pressure [13], for $\mathrm{RbCuF}_{3}$ [14], and $\left(\mathrm{NH}_{4}\right) \mathrm{CuF}_{3}$ [15], compared to our calculations and the values obtained for constant short $\mathrm{Cu}-\mathrm{F}$ distance $s_{\min }$.

The mechanism driving the distortion $\delta$ and orbital ordering has been the subject of intense controversy. As early as 1960, Kanamori noted that the structure of $\mathrm{KCuF}_{3}$ could arise from the cooperative Jahn-Teller effect [2]. Later, Kugel and Khomskii showed that orbital order in $\mathrm{KCuF}_{3}$ can originate from electronic superexchange even in the absence of distortions [3]. As one of the first applications of the density functional theory plus $U(\mathrm{DFT}+U)$ method [9], Liechtenstein et al. found that a Hubbard $U$ is necessary to stabilize the distorted structure and concluded that an electronic Kugel-Khomskii mechanism drives the transition. In the same year, Towler et al. found that Hartree-Fock also gives reasonable agreement with experiment, despite the complete lack of correlations [10]. Eventually, the question was settled by density functional theory plus dynamical meanfield theory (DFT+DMFT) calculations, which showed that Kugel-Khomskii superexchange alone can only account for orbital order below $T_{\mathrm{KK}} \approx 350 \mathrm{~K}$ [11], while experimentally it persists to much higher temperatures [8]. In fact, the transition to the undistorted high-temperature phase has never been seen experimentally, and the analysis of the crystal structure up to $900 \mathrm{~K}$ showed that contrary to conventional wisdom, the distortion increases with temperature instead of vanishing above some critical value [12]. Applying hydrostatic pressure dramatically reduces the distortion as the lattice constant decreases [13], while expanding the lattice by chemical pressure, substituting $\mathrm{K}$ by $\mathrm{Rb}$ [14] or $\mathrm{NH}_{4}$ [15], results in an increase of the distortion parameter $\delta$ following the same trend as in thermally expanded $\mathrm{KCuF}_{3}$. All this points to the lattice constant, hence the energy rather than entropy, as the key player in determining the size of the distortion (see Fig. 2).

To understand the role of the lattice, we have performed DFT $+U$ calculations for $\mathrm{KCuF}_{3}$ in the unit cell shown in Fig. 1 with the experimental lattice constants at different temperatures. Calculations were performed using the Vienna ab initio Simulation Package (VASP) [16] within the generalized gradient approximation (GGA) of Perdew, Burke, and Ernzerhof [17] to density functional theory using the projector

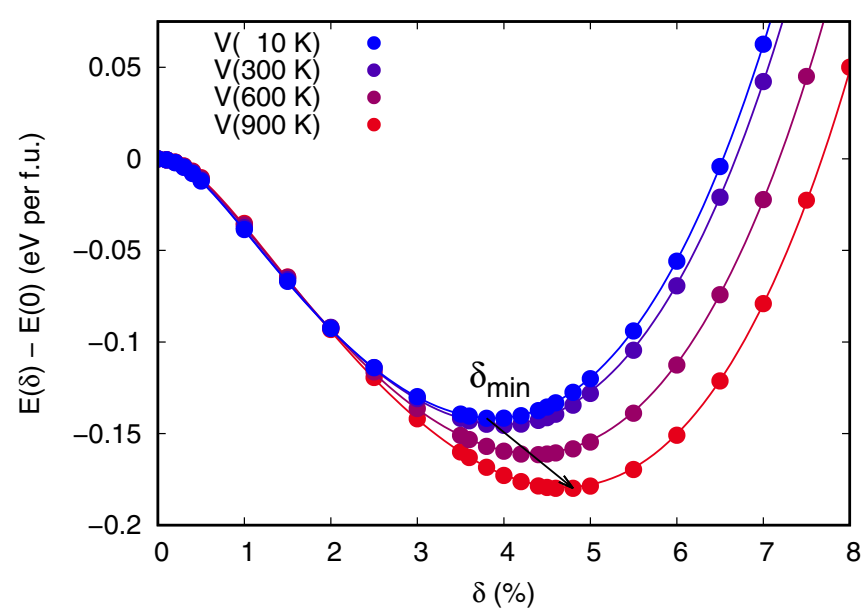

FIG. 3. DFT $+U$ gain in energy per formula unit as a function of the distortion parameter $\delta$ for experimental unit cells at different temperatures. With thermal expansion, the minimum of the energy curve moves to larger distortions $\delta_{\min }$ and deepens. Lines are fits to guide the eye.

augmented-wave (PAW) [18] pseudopotentials of Kresse and Joubert [19]. We apply on-site Coulomb interactions on the $\mathrm{Cu} 3 d$ orbitals through the " $+U$ " correction of Liechtenstein et al. [9] with double-counting corrections in the fully localized limit.

It is known that $\mathrm{DFT}+U$ describes structural properties remarkably well [20]. We also find that the energy gained by moving the fluorine ions agrees with both experimental estimates [21] and calculations explicitly including many-body effects [22,23]. Moreover, extracting the frequency of the $A_{1 g}$ mode, we find excellent agreement with Raman data [24]. Our results are fairly independent of the model parameters $U$ and $J$, as long as they are large enough to open a gap. The main effect of increasing $U$ is to slightly increase the effective radius of the cation [25]. Figure 3 shows the energy gained by distorting the lattice for the experimental unit-cell parameters at increasing temperatures $[12,26]$ calculated using the established values $U=7 \mathrm{eV}$ and $J=0.9 \mathrm{eV}$ [9]. We find that the distortion $\delta$ increases with lattice constant, in good agreement with the experimental values (see Fig. 2).

While our calculations reproduce the observed increase of the distortion very well, neither of the established theoretical mechanisms can explain it: The orbital superexchange coupling decreases with distance so that the Kugel-Khomskii mechanism weakens as the lattice expands [3]. The JahnTeller mechanism could, in principle, explain a distortion that increases with volume. Writing the energy gained by displacing the fluorine ions by $\Delta=(\ell-s) / 2=a \delta / \sqrt{2}$ from their symmetric position as $E_{\mathrm{JT}}(\Delta)=-g \Delta+C \Delta^{2} / 2$, where $g$ gives the splitting of the $e_{g}$ level and $C$ the elastic constant, the energy is minimized for $\Delta_{\mathrm{JT}}=g / C$ [2]. The dependence of $\Delta_{\mathrm{JT}}$ on the lattice constant $a$ is thus given by the change of $g$ and $C$. Both will decrease with the lattice constant, and if the Jahn-Teller mode softens much faster than the electron-phonon coupling weakens, $\Delta_{\mathrm{JT}}$ could become arbitrarily large. From crystal-field theory, we know that $g$ scales with $1 / a^{4}$. To obtain the experimentally observed increase in $\Delta_{\min }$, the 


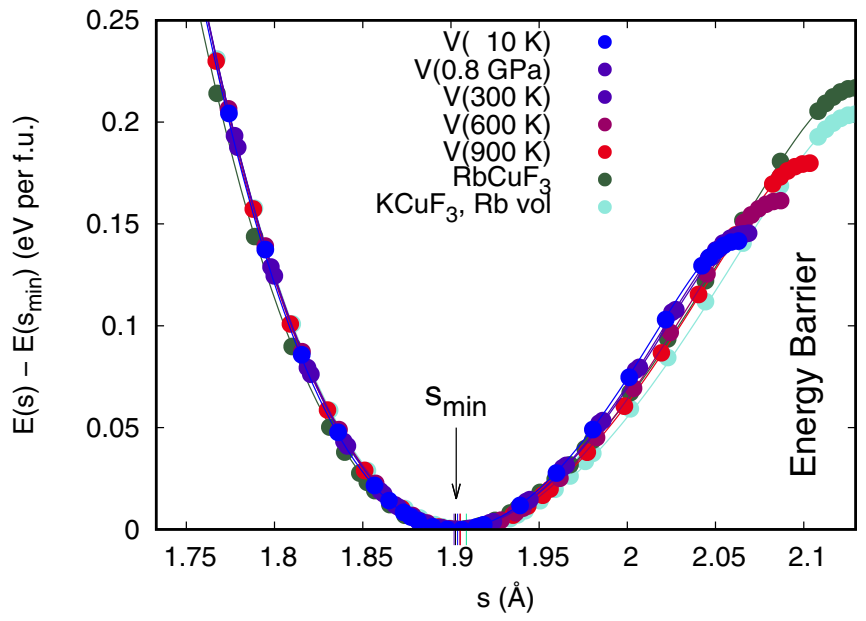

FIG. 4. Change in DFT $+U$ energy as a function of $\mathrm{Cu}-\mathrm{F}$ distance $s$ for different lattice constants $a$. For $s$ smaller than $s_{\min }$, the energy curves are practically independent of the actual lattice. For $s$ larger than $s_{\min }$, each curve reaches a maximum at the undistorted position $s=a / 2 \sqrt{2}$.

elastic constant $C$ would have to decay faster than $1 / a^{14}$. This contradicts, however, the observed temperature (volume) dependence of the Jahn-Teller mode ( $A_{1 g}$ in [24]), ruling out the Jahn-Teller mechanism as well. Thus the two established mechanisms completely fail to explain the observed increase in the order parameter with temperature.

The complete failure of the established distortion mechanisms calls for a change of perspective. Instead of focusing on the displacement from the high-symmetry position, we consider the $\mathrm{Cu}-\mathrm{F}$ distance. The shortest distance $s$ should be given by when the ions touch. In fact, for $\mathrm{KCuF}_{3}$, it is practically independent of temperature [12] so that the increase of $\Delta$ is simply a consequence of thermal expansion. The same is true when applying pressure [13], substituting $\mathrm{K}$ by $\mathrm{Rb}$ [14], or $\mathrm{NH}_{4}$ [15]. We can make this picture quantitative by plotting the energy curves of Fig. 3 as a function of the Cu-F distance (see Fig. 4): pushing the ions closer together than the optimal distance results in a strong repulsion-a Born-Mayer potential [27].

This suggests a different model: we describe $E(\Delta)$ using a Born-Mayer repulsion energy $E_{\mathrm{BM}}=A \exp \left(-r_{\mathrm{Cu}-\mathrm{F}} / \rho\right)$, the Ewald energy $E_{\text {Ewald }}$ of the periodic arrangement of ions, and a term $E_{\mathrm{CF}}=\Gamma-\sqrt{\Gamma^{2}+(g \Delta)^{2}}$ describing the crystal-field splitting of the $e_{g}$ level due to the tetragonal compression and the displacement $\Delta$. Since the ionic charges are practically independent of the lattice constant, as are the Born-Mayer coefficients $A$ and $\rho$ (that also describe the bond length of the $\mathrm{CuF}_{2}$ molecule correctly), the couplings $\Gamma$ and $g$ are the only parameters that depend on the lattice constant. As expected, the crystal-field splitting scales as $1 / a^{4}$, while the change in Ewald energy can be approximated by $-C_{\text {Ewald }} \Delta^{2} / 2$, where $C_{\text {Ewald }}$ scales as $1 / a^{3}$, as it should. The resulting expression,

$$
\begin{aligned}
E_{\text {ionJT }}(\Delta)= & \Gamma-\sqrt{\Gamma^{2}+(g \Delta)^{2}}-C_{\text {Ewald }} \Delta^{2} / 2 \\
& +2 B e^{-a / \rho 2 \sqrt{2}}[\cosh (\Delta / \rho)-1],
\end{aligned}
$$

gives not only excellent fits to the DFT $+U$ energies for $\mathrm{KCuF}_{3}$, as shown by the curves in Figs. 3 and 4 with the parameters
TABLE I. Model parameters for $E_{\text {ionTT }}(\Delta)$ that fit the DFT $+U$ curves calculated for $\mathrm{KCuF}_{3}$ with experimental lattice constants at different temperatures (Figs. 3 and 4). $\Gamma$ increases with the tetragonal distortion, while $g$ decreases with $a$. The Born-Mayer parameters $B=$ $9188 \mathrm{eV}$ and $\rho=0.2186 \AA$ are independent of the lattice constants. This is also true for the charges of the ions entering the Ewald energy: $Z_{\mathrm{Cu}}=1.86 e=2 Z_{\mathrm{K}}=-2 Z_{\mathrm{F}}$.

\begin{tabular}{lccccc}
\hline \hline$T(\mathrm{~K})$ & $a(\AA)$ & $c(\AA)$ & $\Gamma(\mathrm{eV})$ & $g(\mathrm{eV} / \AA)$ & $C_{\text {Ewald }}\left(\mathrm{eV} / \AA^{2}\right)$ \\
\hline 10 & 5.835 & 7.828 & 0.0620 & 2.195 & 20.059 \\
300 & 5.852 & 7.841 & 0.0640 & 2.173 & 19.877 \\
600 & 5.903 & 7.897 & 0.0677 & 2.112 & 19.342 \\
900 & 5.950 & 7.954 & 0.0698 & 2.060 & 18.883 \\
\hline \hline
\end{tabular}

given in Table I, but should also describe strongly ionic JahnTeller-active compounds in general.

We can now explain the anomalous behavior seen in $\mathrm{KCuF}_{3}$. To simplify the discussion, we neglect for the moment the tetragonal splitting, setting $\Gamma=0$. The $\mathrm{Cu}^{2+}$ cation is fairly small, i.e., $\rho \ll a / 2 \sqrt{2}$, so that the frequency about the undistorted position, $m \omega_{0}^{2}=d^{2} E(\Delta) /\left.d \Delta^{2}\right|_{\Delta=0}=$ $2 B e^{-a / \rho 2 \sqrt{2}} / \rho^{2}-C_{\text {Ewald }}$, is quite low. In a simple Jahn-Teller picture, this would imply a very large distortion $\Delta_{\mathrm{JT}}$, which would bring $\mathrm{Cu}$ and $\mathrm{F}$ extremely close to each other. In reality, however, the ions repel strongly at short distance. Since this Born-Mayer repulsion increases exponentially, the distortion will be stopped at a $\mathrm{Cu}-\mathrm{F}$ distance $s_{\min }$ that is practically independent of the lattice constant. The observed linear increase of the distortion with the lattice constant $\Delta_{\min }(a) \approx$ $a / 2 \sqrt{2}-s_{\min }$ is thus simply the consequence of a constant $s_{\min }$ (see the line in Fig. 2). At the same time, the energy gained from the distortion increases with $\Delta_{\min }$. The thermal expansion $a(T)$ thus stabilizes the distortion in $\mathrm{KCuF}_{3}$, explaining the absence of a transition to the undistorted structure. We note that in our model, the frequency $\omega_{0}$ differs from the frequency of the $A_{1 g}$ Raman mode, which is given by the expansion about the minimum: $m \omega_{A_{1 g}}^{2}=d^{2} E(\Delta) /\left.d \Delta^{2}\right|_{\Delta=\Delta_{\min }}$. The difference is due to the Born-Mayer potential, which makes the $A_{1 g}$ mode quite anharmonic, in agreement with experiment [24]. The scenario of fixed $s_{\min }$ is not limited to $\mathrm{KCuF}_{3}$. In fact, Table 5 of Ref. [14] lists the short Cu-F distances $s$ of 13 materials of widely varying structure and composition with Jahn-Teller-active $\mathrm{CuF}_{6}$ octahedra. They all differ by less than $2 \%$.

For larger cations, $\omega_{0}$ will be harder, leading to smaller distortions and a more Jahn-Teller-like picture. There is, however, a crucial difference: $\omega_{0}$ softens dramatically with the expansion of the lattice, leading to a robust distortion even as the temperature increases. Remarkably, this is what is actually observed in the tetragonal phase of another fluoride,

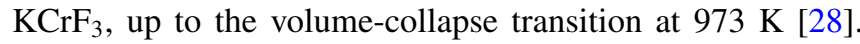
This behavior is markedly different from that typical of the more covalent transition-metal oxides, e.g., $\mathrm{LaMnO}_{3}$, where the distortion decreases gradually with $T$ until it vanishes at the transition temperature [29]. The crucial difference is whether the Jahn-Teller distortion is so large that neighboring atoms touch. In that case, the distortion will be halted by the Born-Mayer repulsion and it will thus increase as the 


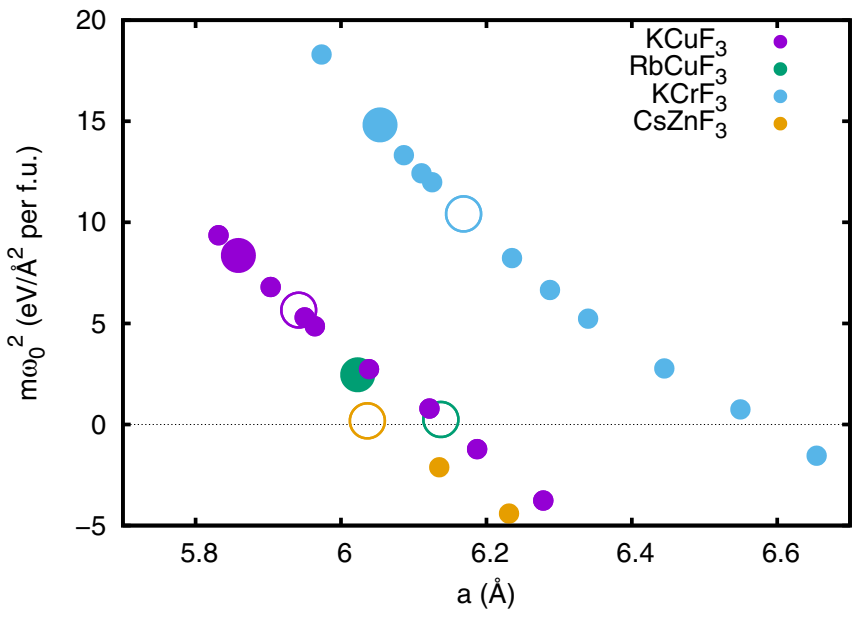

FIG. 5. Dependence of the calculated elastic constant $m \omega_{0}^{2}$ for the distortion about $\Delta=0$ on the lattice parameter $a$. For the open-shell systems, the values are calculated by DFT $+U$ with $U=7 \mathrm{eV}$ and $J=0.9 \mathrm{eV}$ for $\mathrm{KCuF}_{3}$ and $\mathrm{RbCuF}_{3}$, and $U=6 \mathrm{eV}$ and $J=0.9 \mathrm{eV}$ for $\mathrm{KCrF}_{3}$, while the closed-shell $\mathrm{CsZnF}_{3}$ is already gapped without a Hubbard $U$. Large filled circles indicate the experimental lattice constant at room temperature; open circles indicate the DFT $+U$ or DFT relaxed lattice constant, which are between 1-2\% larger than the experimental values. For all compounds, the elastic constant changes sign when the lattice constant gets large enough. The larger the cation, the larger the critical lattice constant $a_{c}$. For the smallest, $\mathrm{Zn}$, the relaxed structure is tantalizingly close to the value required for an inverted Landau transition.

lattice expands. The resulting persistence of the distortion with increasing temperature can be expected for all JahnTeller-active compounds with significant thermal expansion coefficients and rapidly softening Jahn-Teller mode, as is common in ionic compounds. This thermally assisted ordering mechanism should be particularly useful for engineering materials, where we want the symmetry-broken phase to survive to high temperatures [30]. Moreover, it suggests an intriguing scenario: When $a$ exceeds the critical value $a_{c}=\rho 2 \sqrt{2} \ln \left[2 B / \rho^{2} C_{\text {Ewald }}\left(a_{c}\right)\right]$, the frequency $\omega_{0}$ becomes imaginary so that even a system with a non-Jahn-Teller-active cation would start to distort. When $a_{c}$ is crossed in thermal expansion, such a system could show an inverted Landau transition from a high-symmetry phase at low temperatures to an ordered high-temperature structure. Ideal candidates are compounds with large lattice constant and small B-site cation, as shown in Fig. 5. While it might be difficult to find a material where $a_{c}$ can be reached by thermal expansion alone, it is conceivable to additionally increase the lattice constant by strain [31] or negative pressure [32] to just beyond the critical value, so that this unusual phase transition can be reached.

It turns out, then, that Landau theory of structural transitions is oversimplified in that it assumes a temperature-independent electronic Hamiltonian, i.e., energy, while the system disorders due to the phonon entropy of the distortion mode [6]. We have identified a striking example which highlights the failure of this standard model of symmetry breaking: in the paradigmatic orbitally ordered perovskite $\mathrm{KCuF}_{3}$, order is stabilized by thermal expansion. Such a behavior naturally results when the distortion is stopped by the Born-Mayer repulsion of the ions. This mechanism will be dominant in particular in an ionic system, but is also active in covalent orbitally ordered materials with soft Jahn-Teller mode. In fact, this is just an instance of a more general principle: When the effective Hamiltonian describing symmetry breaking has a significant temperature dependence, we can expect to observe more exotic phenomena than predicted by Landau theory.

This work has been supported by the Deutsche Forschungsgemeinschaft through FOR 1346. Calculations have been done on JURECA at the Jülich Supercomputer Centre under Grant No. GRS300.
[1] Y. Tokura and N. Nagaosa, Science 288, 462 (2000).

[2] J. Kanamori, J. Appl. Phys. 31, S14 (1960).

[3] K. I. Kugel' and D. I. Khomskii, Zh. Eksp. Teor. Fiz. 64, 1429 (1973) [Sov. Phys. JETP 37, 725 (1973)].

[4] D. I. Khomskii, Transition Metal Compounds (Cambridge University Press, Cambridge, 2014).

[5] J.-C. Tolédano and P. Tolédano, The Landau Theory of Phase Transitions (World Scientific, Singapore, 1987).

[6] M. C. Dove, Structure and Dynamics (Oxford University Press, Oxford, 2003).

[7] I. B. Bersuker, The Jahn-Teller Effect (Cambridge University Press, Cambridge, 2006).

[8] A. Okazaki and Y. Suemune, J. Phys. Soc. Jpn. 16, 176 (1961).

[9] A. I. Liechtenstein, V. I. Anisimov, and J. Zaanen, Phys. Rev. B 52, R5467(R) (1995).

[10] M. D. Towler, R. Dovesi, and V. R. Saunders, Phys. Rev. B 52, 10150 (1995).

[11] E. Pavarini, E. Koch, and A. I. Lichtenstein, Phys. Rev. Lett. 101, 266405 (2008).
[12] L. G. Marshall, J. Zhou, J. Zhang, J. Han, S. C. Vogel, X. Yu, Y. Zhao, M. T. Fernández-Díaz, J. Cheng, and J. B. Goodenough, Phys. Rev. B 87, 014109 (2013).

[13] J.-S. Zhou, J. A. Alonso, J. T. Han, M. T. Fernández-Díaz, J.-G. Cheng, and J. B. Goodenough, J. Fluorine Chem. 132, 1117 (2011).

[14] V. Kaiser, M. Otto, F. Binder, and D. Babel, Z. Chem. 585, 93 (1990).

[15] S. I. Troyanov, I. V. Morozov, and Y. M. Korenev, Zh. Neorg. Khim. 38, 984 (1993) [Russian J. Inorg. Chem. 38, 909 (1993)].

[16] G. Kresse and J. Furthmüller, Comput. Mater. Sci. 6, 15 (1996).

[17] J. P. Perdew, K. Burke, and M. Ernzerhof, Phys. Rev. Lett. 77, 3865 (1996).

[18] P. E. Blöchl, Phys. Rev. B 50, 17953 (1994).

[19] G. Kresse and D. Joubert, Phys. Rev. B 59, 1758 (1999).

[20] N. Binggeli and M. Altarelli, Phys. Rev. B 70, 085117 (2004).

[21] P. Ghigna, M. Scavini, C. Mazzoli, M. Brunelli, C. Laurenti, and C. Ferrero, Phys. Rev. B 81, 073107 (2010). 
[22] I. Leonov, N. Binggeli, D. Korotin, V. I. Anisimov, N. Stojić, and D. Vollhardt, Phys. Rev. Lett. 101, 096405 (2008).

[23] A. Flesch, G. Zhang, E. Koch, and E. Pavarini, Phys. Rev. B 85, 035124 (2012).

[24] J. C. T. Lee, S. Yuan, S. Lal, Y. E. Joe, Y. Gan, S. Samadici, K. Finkelstein, Y. Feng, A. Rusydi, P. M. Goldbart, S. L. Cooper, and P. Abbamonte, Nat. Phys. 8, 63 (2012).

[25] C. Autieri, E. Koch, and E. Pavarini, Phys. Rev. B 89, 155109 (2014).

[26] S. K. Satija, J. D. Axe, G. Shirane, H. Yoshizawa, and K. Hirakawa, Phys. Rev. B 21, 2001 (1980).

[27] M. Born and J. E. Mayer, Z. Phys. 75, 1 (1932).
[28] S. Margadonna and G. Karotsis, J. Mater. Chem. 17, 2013 (2007).

[29] J. Rodriguez-Carvajal, M. Hennion, F. Moussa, A. H. Moudden, L. Pinsard, and A. Revcolevschi, Phys. Rev. B 57, R3189(R) (1998).

[30] J. B. Goodenough, Chem. Mater. 26, 820 (2013).

[31] J. H. Haeni, P. Irvin, W. Chang, R. Uecker, P. Reiche, U. L. Li, S. Choudhury, W. Tian, M. E. Hawley, B. Craigo, A. K. Tagantsev, X. Q. Pan, S. K. Streiffer, L. Q. Chen, S. W. Kirchoefer, J. Levy, and D. G. Schlom, Nature (London) 430, 758 (2004).

[32] J. Wang, B. Wylie-van Eerd, T. Sluka, C. Sandu, M. Cantoni, X.-K. Wei, A. Kvasov, L. J. McGilly, P. Gemeiner, B. Dkhil, A. Tagantsev, J. Trodahl, and N. Setter, Nat. Mater. 14, 985 (2015). 